\title{
ScRNA-seq revealed the kinetic of nasopharyngeal immune responses in asymptomatic COVID-19 carriers
}

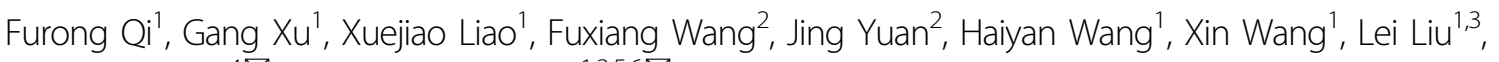 \\ Shuye Zhang $\mathbb{1}^{4 凶}$ and Zheng Zhang $\mathbb{1}^{1,3,5,6 \times}$
}

Dear Editor,

Asymptomatic (or silent) severe acute respiratory syndrome-coronavirus-2 (SARS-CoV-2) infections are unique and curtail the control of viral transmission; however, it is unclear how the immune system of asymptomatic carriers reacts particularly when it comes to clearing the virus and sparing the host from any symptoms. Here, we revealed the kinetics of nasopharyngeal immune responses in asymptomatic carriers using single-cell transcriptomic RNA sequencing (scRNA-seq). A unique immune homeostatic environment was found in the nasopharynx locally in asymptomatic infections, including strengthening of epithelial compartments, sequential transition of monocyte/ macrophage functions, vigorous $\mathrm{CD}^{+} \mathrm{T}$-cell clonal expansion. This supports that a rapid and coordinated immune defense at the respiratory barrier site is the key to the successful control of SARS-CoV-2 infections.

The coronavirus disease 2019 (COVID-19) pandemic is one of the most serious public health crises in human history. The clinical manifestations of COVID-19 are diverse, ranging from asymptomatic infections, mild symptoms, to viral pneumonia and life-threatening acute respiratory distress syndrome ${ }^{1}$. Asymptomatic infections, such as patients who are infected with SARS-CoV-2 but show no symptoms, are very common but hard to

\footnotetext{
Correspondence: Shuye Zhang (zhangshuye@shphc.org.cn) or

Zheng Zhang (zhangzheng1975@aliyun.com)

'Institute for Hepatology, National Clinical Research Center for Infectious

Disease, Shenzhen Third People's Hospital; The Second Affiliated Hospital,

School of Medicine, Southern University of Science and Technology, Shenzhen,

Guangdong, China

2Department for Infectious Diseases, Shenzhen Third People's Hospital,

Shenzhen, Guangdong, China

Full list of author information is available at the end of the article

These authors contributed equally: Furong Qi, Gang Xu, Xuejiao Liao
}

identify, making efforts to stop transmission almost impossible ${ }^{2,3}$. Although previous studies have revealed some aspects of immune responses in symptomatic COVID-19 patients ${ }^{4,5}$, little is known about the immune responses especially in the upper respiratory mucosal barriers where the SARS-CoV-2 infection starts.

In this study, scRNA-seq was used to profile the nasopharyngeal swabs of five asymptomatic COVID-19 carriers, at both the early-stage (AA, within 3 days after PCR positivity confirmation) and late-stage (AP, within 6 days after PCR turned negative) of the infection. The publicly available scRNA-seq data of nasopharyngeal swabs from 8 mild $(\mathrm{M})$ and 11 severe patients (S) and 6 healthy controls (H) were also evaluated ${ }^{6}$ (Fig. 1a). In total, 97,251 cells (Supplementary Fig. S1a and Table S1) passed the stringent quality control and were categorized into 9 cell lineages, including epithelial cells (club, ciliated, and squamous), $\mathrm{T}$ cells (CD8-GZMB and CD8-RPS27), B cells, neutrophils, and macrophages (M $\varphi$-FCN1 and $\mathrm{M} \varphi$ APOE) based on their specific markers (Fig. 1b).

Of the five enrolled asymptomatic carriers, none selfreported any symptoms, which was also verified by the examining results of the common clinical manifestations. All clinical endpoints throughout the study displayed nearnormal values, including C-Reactive Protein (CRP), interleukin 6 (IL-6), lactate dehydrogenase (LDH), D-dimer, and blood cell counts (Supplementary Table S2). There were low levels of viral RNA (high Ct values) in the earlystage of asymptomatic infection, as identified through nasopharyngeal swabs, whereas viral RNA turned negative in the late-stage samples. Examining the scRNA-seq data, we also found that viral RNA was undetectable in asymptomatic cases (Supplementary Fig. S1b), possibly due to the low viral load in these carriers. By contrast, the 


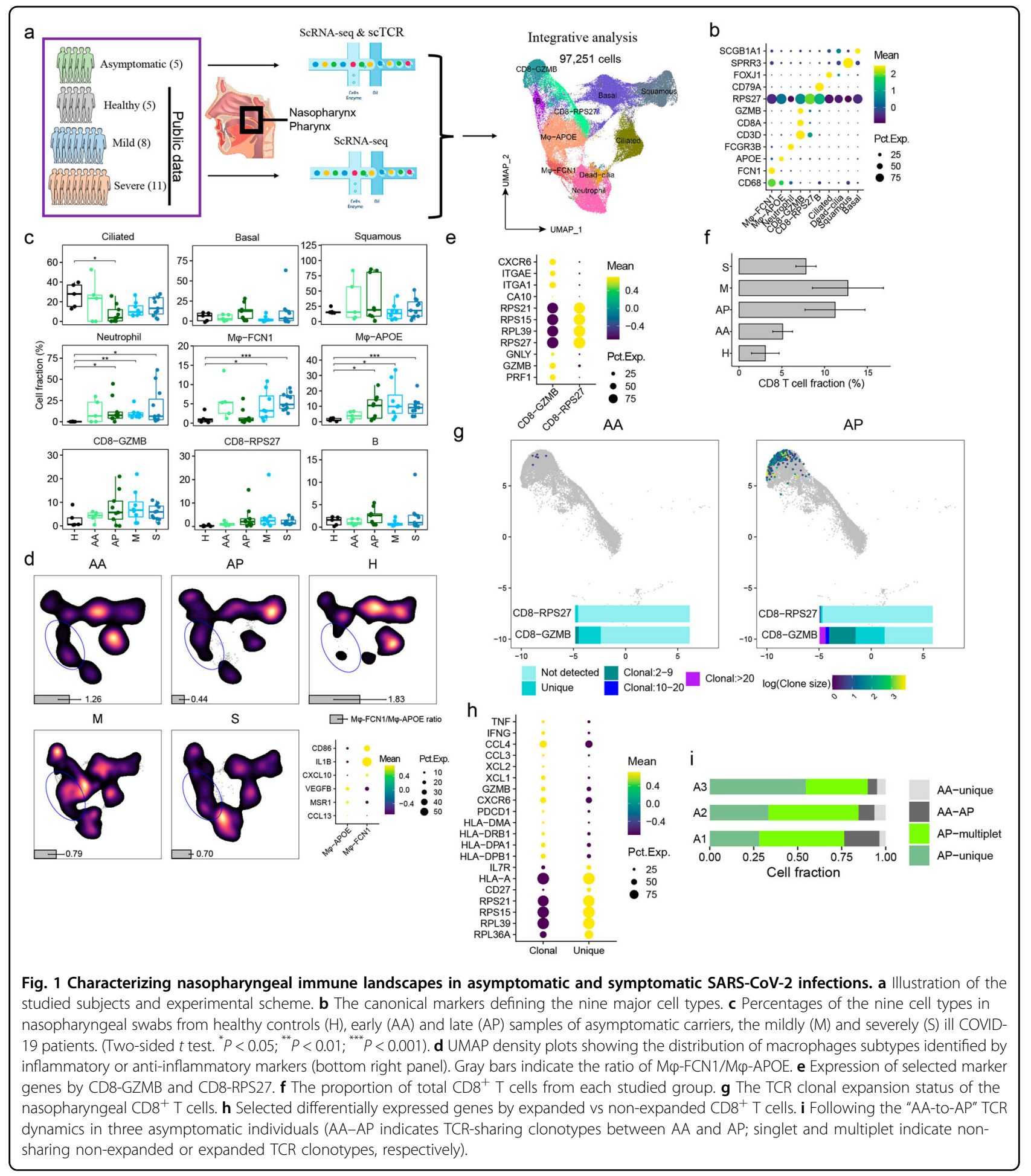

viral RNA was present in swabs from mild and severe COVID-19 patients (Supplementary Fig. S1c).

Epithelium is the first barrier against invading pathogens $^{7}$. It is unclear, however, what roles epithelial cells play in the defense against SARS-CoV-2 infections. The key host genes $A C E 2^{8}, T M R P S S 2^{9}, N R P 1^{10}$, and $N R P 2$, which mediate SARS-CoV-2 infection, are expressed by epithelial cells and upregulated in those with symptomatic vs asymptomatic infections (Supplementary Fig. S1c, d). Three types of epithelial cells were identified and analyzed in this study. They have an intriguing division of labor. For example, the genes related to immune responses 
including neutrophil degranulation and NF- $\mathrm{KB}$ signaling (ECM1, IL1RN, NDFIP2, NFKBIA, etc.), were specifically enriched in squamous cells (Supplementary Fig. S1e, f). The ciliated cells show signatures of cilium-related functions, and Basal cells, as potential progenitors, show metabolism-related signatures (Supplementary Fig. S1e, f). Both ciliated and basal cells were decreased in COVID-19 patients (Fig. 1c). Notably, the barrier functions of ciliated (cilium assembly) and squamous cells (epiderm differentiation and cytokine production) were strengthened in asymptomatic infections, while there was an increase of type I IFN-activated signature in symptomatic infections (Supplementary Fig. S1g).

Besides the epithelium remodeling, the nasopharyngeal immune cellular compartments in asymptomatic carriers differed significantly from those in healthy controls and symptomatic cases. For example, there was increased infiltration of immune cells, including neutrophils, macrophages, and $\mathrm{T}$ lymphocytes compared to healthy controls (Fig. 1c). Pronounced neutrophil functionality indicated by higher levels of neutrophil degranulation genes, and IL-1, IL-12, and IFN- $\gamma$ responses were observed in severe COVID-19 patients. In contrast, neutrophil activity was more confined in asymptomatic carriers and mild COVID-19 patients (Supplementary Fig. S2a, b). It is unclear whether the increased infiltration of neutrophils plays any anti-viral roles.

Nasopharyngeal macrophages were also analyzed and consisted of two subtypes: M $\varphi$-FCN1 expresses CXCL10, $I L 1 B$, and $C D 86$ as the pro-inflammatory M1-type, and $\mathrm{M} \varphi$ APOE expresses CCL13, MSR1, and VEGFB as the antiinflammatory M2-type (Fig. 1d). The ratios of M1 and M2 macrophages changed sequentially in asymptomatic infections. The early-stage was dominated by the M1 (1.26-fold of M1/M2 ratio), while the later stage was dominated by the M2 macrophages (0.44-fold of M1/M2 ratio) (Fig. 1d). By analyzing macrophage transcriptomes, it was found that the genes related to the cellular response to lipopolysaccharide and multiple chemokines were highly expressed in AA, implying that innate immunity was primarily activated at the AA stage of viral infection. Whereas the genes involved in macrophage activation and T-cell receptor signaling pathway were elevated in AP (Supplementary Fig. S3a, b). This signifies a transition from pro-inflammatory to tissue-repairing functions in those recovering from an acute respiratory infection. Only low levels of cytokines (IL-6, IL-10, IL-12, and IL-17A) were detected in plasma from asymptomatic patients (Supplementary Fig. S3c and Table S3), in consistence with lower levels of nasopharyngeal inflammation. However, severe patients expressed elevated transcripts related to response to the virus, neutrophil degranulation, response to IL-1 and Fc receptor signaling pathway (Supplementary Fig. S3d). The nasopharyngeal macrophages in those severe
COVID-19 patients produced higher levels of chemokines (CCL2, CCL3, CCL4, CXCL1, CXCL2, CXCL3), indicating their roles in maintaining the cytokine storm (Supplementary Fig. S3e).

We also found two subsets of nasopharyngeal $\mathrm{T}$ cells, the CD8-GZMB and CD8-RPS27. CD8-GZMB subset expressed tissue-residency markers CXCR6, ITGA1, $I T G A E$, and effector genes GZMB, GNLY, PRF1, therefore identifying them as the tissue-resident $\mathrm{T}$ cells with effector functions. And the CD8-RPS27 subset highly expressed ribosomal genes but lacked typical effector molecules (Supplementary Fig. S4a and Fig. 1e). We found increased nasopharyngeal $\mathrm{CD}^{+} \mathrm{T}$ cells infiltrates from early to late-stage of asymptomatic infections (Fig. 1f). ScTCR analysis revealed much higher clonal expansion of CD8-GZMB cells in AP versus AA (Fig. 1g), suggesting that viral infection triggered an adaptive immune response at the AP stage. Clonally expanded $\mathrm{CD}^{+} \mathrm{T}$ cells expressed higher levels of IFNG, TNF, HLA-DR, and CXCR6 (Fig. 1h) than their non-clonal counterparts, supporting that expanded nasopharyngeal CD8-GZMB cell clones reacted against SARS-CoV-2 infection. Examining the sequential samples individually, newly clone-expanded CD8-GZMB T-cell clones (with unique, non-sharing TCR clonality) were continuously present at the mucosal barriers during asymptomatic infections (Fig. 1i), probably as the key reinforcement to clear the virus and strengthen the barrier defense.

Finally, to further understand the nasopharyngeal immune microenvironment in asymptomatic COVID-19, the cell-cell interaction network was examined through analyzing receptor-ligand pairs among squamous, neutrophils, macrophages, and CD8-GZMB cells. Healthy controls were omitted due to the scarcity of immune cells. Macrophage cross-talking with other cells is the most prominent, and severe patients distinguished other cases by the enhanced neutrophil crosstalk (Supplementary Fig. S4b). Several signaling partners, including multiple chemokine receptors (CXCL1/3-CXCR1/2, CCL7-CCR1, CCL3$C C R 1)$, are specifically upregulated between macrophages and neutrophils in patients experiencing severe COVID-19 (Supplementary Fig. S4c). In contrast, the enhanced interactions between squamous epithelial cells and macrophages in asymptomatic individuals were identified, including MIF-CD74, IL1RN-IL1R, AREGICAM1, and TNF/CCL4L2-VSIR (Supplementary Fig. S4d). These cells have been previously reported to participate in immune inhibition and repair, suggesting a unique immune homeostatic environment at mucosal barriers during asymptomatic SARS-CoV-2 infection.

In summary, this study provides the first comprehensive transcriptional and cellular landscape of the nasopharyngeal mucosa in asymptomatic COVID-19 carriers. These findings highlight an optimal immune response effectively 
clearing the virus without causing diseases, characterized by enhanced epithelium barrier function, mild inflammation, and robust $\mathrm{CD}^{+}{ }^{+}$-cell response locally.

\section{Acknowledgements}

We acknowledge the work and contribution of all the health providers from Shenzhen Third People's Hospital. We also thank patients for their active participation. This study was supported by the National Science Fund for Distinguished Young Scholars (82025022), Science and Technology Innovation Committee of Shenzhen Municipality (2020A1111350032), Bill \& Melinda Gates Foundation, Central Charity Fund of Chinese Academy of Medical Science (2020-PT310-009), and the Natural Science Foundation of Guangdong Province of China (2020B1111340027, 2020B1111340039) and Shenzhen Bay Funding (2020B1111340075). The funders had no role in study design, data collection, data analysis, data interpretation, or writing of the report.

\section{Author details}

${ }^{1}$ Institute for Hepatology, National Clinical Research Center for Infectious Disease, Shenzhen Third People's Hospital; The Second Affiliated Hospital, School of Medicine, Southern University of Science and Technology, Shenzhen, Guangdong, China. ${ }^{2}$ Department for Infectious Diseases, Shenzhen Third People's Hospital, Shenzhen, Guangdong, China. ${ }^{3}$ Shenzhen Research Center for Communicable Disease Diagnosis and Treatment of Chinese Academy of Medical Science, Shenzhen, Guangdong, China. ${ }^{4}$ Shanghai Public Health Clinical Center, Fudan University, Shanghai, China. ${ }^{5}$ Shenzhen Bay Laboratory, Shenzhen, Guangdong, China. 'Guangdong Key Laboratory for Anti-infection Drug Quality Evaluation, Shenzhen, Guangdong, China

\section{Author contributions}

S.Z. and Z.Z. designed this study and wrote the manuscript. F.Q. and G.X performed this study and wrote the manuscript. F.Q. performed the bioinformatics analysis. G.X. performed the experiments. X.L. collected the specimens and provided the patients' clinical information. F.W., J.Y., H.W., X.W. and L.L. revised the manuscript.

\section{Conflict of interest}

The authors declare no competing interests.

\section{Publisher's note}

Springer Nature remains neutral with regard to jurisdictional claims in published maps and institutional affiliations.

Supplementary information The online version contains supplementary material available at https://doi.org/10.1038/s41421-021-00294-x.

Received: 4 February 2021 Accepted: 17 June 2021

Published online: 27 July 2021

\section{References}

1. Hu, B., Guo, H., Zhou, P. \& Shi, Z.L. Characteristics of SARS-CoV-2 and COVID-19. Nat. Rev. Microbiol. 19, 141-154 (2021).

2. Nikolai, L. A., Meyer, C. G., Kremsner, P. G. \& Velavan, T. P. Asymptomatic SARS Coronavirus 2 infection: invisible yet invincible. Int. J. Infect. Dis. 100, 112-116 (2020).

3. Zhao, D. et al. Asymptomatic infection by SARS-CoV-2 in healthcare workers: a study in a large teaching hospital in Wuhan, China. Int. J. Infect. Dis. 99, 219-225 (2020).

4. Long, Q. X. et al. Clinical and immunological assessment of asymptomatic SARS-CoV-2 infections. Nat. Med. 26, 1200-1204 (2020).

5. Stefano, G. B. \& Kream, R. M. Convalescent memory T cell immunity in individuals with mild or asymptomatic SARS-CoV-2 infection may result from an evolutionarily adapted immune response to coronavirus and the 'common cold'. Med. Sci. Monit. 26, e929789 (2020).

6. Chua, R. L. et al. COVID-19 severity correlates with airway epithelium-immune cell interactions identified by single-cell analysis. Nat. Biotechnol. 38, 970-979 (2020).

7. Xiao, C. et al. Defective epithelial barrier function in asthma. J. Allergy Clin. Immunol. 128, 549-556 (2011).

8. Zhou, P. et al. A pneumonia outbreak associated with a new coronavirus of probable bat origin. Nature 579, 270-273 (2020).

9. Hoffmann, M. et al. SARS-CoV-2 cell entry depends on ACE2 and TMPRSS2 and is blocked by a clinically proven protease inhibitor. Cell 181, 271-280 (2020).

10. Daly, J. L. et al. Neuropilin-1 is a host factor for SARS-CoV-2 infection. Science 370, 861-865 (2020). 